\title{
Polymorphism rs I0I05606 of LPL as a Novel Risk Factor for Microalbuminuria
}

\author{
Zhu Wei Lim' \\ Wei Liang Chen (D) ${ }^{2-4}$ \\ 'Department of Obstetrics and \\ Gynecology, Changhua Christian \\ Hospital, Changhua, Taiwan, Republic of \\ China; ${ }^{2}$ Division of Family Medicine, \\ Department of Family and Community \\ Medicine, Tri-Service General Hospital; \\ and School of Medicine, National \\ Defense Medical Center, Taipei, Taiwan, \\ Republic of China; ${ }^{3}$ Division of Geriatric \\ Medicine, Department of Family and \\ Community Medicine, Tri-Service \\ General Hospital; and School of \\ Medicine, National Defense Medical \\ Center, Taipei, Taiwan, Republic of China; \\ ${ }^{4}$ Department of Biochemistry, National \\ Defense Medical Center, Taipei, Taiwan, \\ Republic of China
}

Introduction: An important clinical feature of metabolic syndrome is abdominal obesity. Microalbuminuria is important in predicting the risk of cardiovascular and renal complications in abdominal obesity patients. However, the association between microalbuminuria polymorphism and abdominal obesity has not been conducted. The objective of this study is to analyze the genetic polymorphism of microalbuminuria in participants with metabolically unhealthy obesity (MUO).

Methods: Among 1325 MUO participants, we identified genomic loci underlying those with microalbuminuria, compared to those without microalbuminuria. Single nucleotide polymorphisms (SNPs) were selected with $\mathrm{P}<1 \times 10^{-5}$ from the Manhattan plot. Multivariable linear regression and analysis of variance were used to analyze the association between different SNP genotypes and microalbuminuria.

Results: The analysis showed homozygous participants for the risk allele A of rs10105606 and Affx-31885823 had 1.978-fold risk and 1.921-fold increased risk of microalbuminuria, respectively. Heterozygous distribution of rs117180252, rs10105606, and Affx-31885823 also increased the risk of microalbuminuria compared to the wild type. Further analysis showed Lipoprotein lipase ( $L P L)$, RN7SL87P, and RPL30P9 were the candidate genes associated with lipid metabolism and abdominal obesity.

Conclusion: In conclusion, LPL, RN7SL87P, and RPL3OP9 minor allele carriers with abdominal obesity are more susceptible to microalbuminuria, explaining the inter-individual differences of microalbuminuria in MUO patients.

Keywords: metabolic syndrome, abdominal obesity, microalbuminuria, metabolically unhealthy obesity, polymorphism, lipoprotein lipase

\section{Introduction}

Metabolic syndrome has emerged as an important public health issue in Taiwan and the worldwide, not only increasing chronic diseases such as cerebrovascular disease, heart disease, diabetes, and hypertension, but also becoming the top ten causes of death in Taiwan every year. According to the Department of Health in Taiwan, a person whose body mass index (BMI) is $27.0 \mathrm{~kg} / \mathrm{m}^{2}$ or higher is considered obese. Metabolically unhealthy obesity (MUO) is defined as abdominal obesity with more than two of metabolic syndrome's components (triglycerides [TGs], high-density lipoprotein cholesterol [HDL-C], systolic blood pressure, and fasting plasma glucose) in the revised National Cholesterol Education Program-Adult Treatment Panel III criteria. ${ }^{1}$

Previous studies have investigated the polymorphisms associated with the components of metabolic syndrome. ${ }^{2}$ In EPIC-NL study, SNPs involved in the 
insulin resistance (PPARG, IRS1, GCKR, IGF1 and $G C K)$, weight regulation (FTO and $M C 4 R$ ) and lipid metabolism (APOB, FADS1-2-3, LPL and etc.) which related to metabolic syndrome were studied. ${ }^{3}$ The association of fat mass and obesity-associated (FTO) gene variants and metabolic syndrome have been widely investigated. ${ }^{4-6}$ In an MUO population, a study demonstrated that a higher frequency of the T45T adiponectin gene would higher the risk of developing metabolic syndrome. ${ }^{7}$

Impaired fasting glucose is a prodrome of type II diabetes, and the latter is a well-known risk factor of chronic kidney disease (CKD). Microalbuminuria can be early detected in $\mathrm{CKD}$ patients. Microalbuminuria is defined as a urine albumin-to-creatinine ratio of $\geq 30 \mathrm{mg}$ / $\mathrm{g}$ or moderately increased albuminuria ( $\geq 30 \mathrm{mg} /$ day). ${ }^{8} \mathrm{~A}$ previous study on the FTO gene showed that rs7204609 polymorphism significantly increased the chances for the presence of central obesity and microalbuminuria in type 2 diabetic patients. ${ }^{9}$ However, to date, polymorphisms associated with microalbuminuria and MUO have not been established. The study aimed to find genetic polymorphisms of microalbuminuria in MUO persons.

\section{Materials and Methods Study Population}

Fifteen thousand three hundred participants between the ages of 30 and 70 with no history of cancer from the Taiwan Biobank (TWB) were included. The TWB conducted a hospital-based cohort study, including participants' genotype data and detailed clinical information. One thousand three hundred twenty-five participants with MUO from the $\mathrm{TWB}^{10,11}$ were selected for this study. Anthropometric measures included microalbuminuria, body waist, systolic pressure, diastolic pressure, $\mathrm{HbAlc}$, fasting glucose, total cholesterol, TG, HDL-C, Glutamic Oxaloacetic Transaminase (GOT), creatinine, and uric acid. All reference values were according to the suggestion of the Ministry of Health and Welfare, Taiwan, or the World Health Organization. Other categorical variables included age and sex. All TWB participants provided written informed consent, and the methods used in this study were carried out according to guidelines and regulations approved by the Institutional Review Board of Tri-Service General Hospital.

\section{Study Variables}

A detailed questionnaire form was required for all TWB participants, which contained information on demographic data, personal histories, past medical histories, and cognitive function. Other data access included urine tests, hematology tests, serology tests and virus tests. Microalbuminuria cases were identified by the levels of urine microalbumin greater than $30 \mathrm{mg} / \mathrm{g}$.

\section{Genotyping and Quality Controls}

The National Center for Genome Medicine cooperated with the Thermo Fisher Scientific factory in the United States to design an SNP identification chip exclusively for Han-Chinese in Taiwan. Axiom Genome-Wide TWB Array Plate (TWB chip; Affymetrix Inc, CA, USA) was commissioned by TWB. Whole-genome genotyping performed by this array plate included 653,291 SNPs for HanChinese descendants of Taiwan.

The linkage disequilibrium (LD) and genotype information were released by TWB, which established the Ethics and Governance Council (http://taiwanview.twbio bank.org.tw). We followed genotype quality control for each individual and SNP levels by using Plink software (http://zzz.bwh.harvard.edu/plink/index.shtml). Also, we performed a principal component analysis (PCA) to assess the population stratification. Age, sex, and creatinine levels were included as covariates to calculate the regression coefficients. We only included SNPs with minor allelic frequencies greater than 0.05 and genotype frequencies with a p-value less than $1 \times 10^{-5}$ under Hardy-Weinberg equilibrium (HWE). Eighteen SNPs were selected. The SNPs were determined on the basis of SNP arrays from the HapMap and 1000 Genomes Project databases, useful human genetics resources. The variants selected for genotyping in our analysis were rs6658296, rs72969423, rs117180252, rs13702, rs10105606, Affx-31885823, rs11227229, rs1558861, rs9326246, rs11216126, rs2075290, rs603446, rs3741298, rs2266788, Affx4282911, rs7396835, rs4769329, and rs17231506. STRING database was used to find out the protein-protein interactions between CAMTA1, ASIC4, LPL, EHBP1L1, BUD13, ZPR1, APOA5, SPATA13 and CETP (https:// string-db.org/).

\section{Statistical Analysis}

All analyses were conducted using Statistical Package for the Social Sciences version 18.0. The chi-square $\left(\chi^{2}\right)$ test was used to verify the relationship between microalbuminuria and SNPs. Continuous variables were expressed as mean and standard deviation. Analysis of variance measured differences between continuous variables and 
urinary albumin excretions. Each SNP was determined under HWE at one degree of freedom using the $\chi^{2}$ test. LD among neighboring SNPs was calculated using The Haploview software. Multivariable linear regression adjusting for potential confounding variables (age, sex, and creatinine) was used to compare changes in variables regression coefficients. We considered $\mathrm{p}<0.05$ as statistically significant.

\section{Results}

The demographic and clinical characteristics of study participants are summarized in Table 1. Figure 1 shows the research flowchart. The hazard ratios and population attributable risks associated with the minor allele are listed in Table 2; results for all associated SNPs, including highly suggestive loci with $\mathrm{P}<1 \times 10^{-5}$, are shown in Table 2 . Eighteen SNPs selected according to the Manhattan plot ( $\mathrm{p}$-value $<1 \times 10^{-5}$ ) are presented in Figure 2. Most of these SNPs are located on chromosome 11 (Table 2). Eighteen SNPs in our study had various functions, including intron variant, prime UTR variant, regulatory region variant, intergenic variant, upstream variant, non-coding transcript variant and two with unknown function (https:// www.ncbi.nlm.nih.gov/snp/). The functions of rs13702, rs2266788, and rs17231506 were 3 Prime UTR Variant of $L P L, A P O A 5$, and Upstream Variant of CETP. ${ }^{12-14}$ $L P L$ was the first and second closest gene to rs 10105606 and Affx-31885823. ${ }^{15}$ APOA5 acted as the same closest gene to rs2075290, rs603446, rs3741298, and Affx4282911, which were functioned as Intron Variant of ZPR1. ${ }^{16-19}$

Table I The Characteristics of Study Participants

\begin{tabular}{|l|l|l|l|}
\hline Characteristics (N = 1325) & Distributions & Mean & SD \\
\hline Age (years) & $30-70$ & 53.90 & 9.12 \\
Male (participants) & 331 (25\%) & & \\
Microalbuminuria (mg/L) & $2.10-919.70$ & 40.82 & 97.63 \\
Body waist (cm) & $73.0-148.0$ & 96.09 & 8.60 \\
Systolic pressure (mm Hg) & $84-211$ & 133.93 & 18.33 \\
Diastolic pressure (mm Hg) & $46-123$ & 81.24 & 11.22 \\
HbAlc $(\%)$ & $3.8-12.5$ & 6.27 & 1.08 \\
Fasting glucose (mg/dL) & $74-321$ & 109.07 & 30.17 \\
Total cholesterol (mg/dL) & $98-507$ & 201.11 & 39.17 \\
Triglyceride (mg/dL) & $39-1817$ & 190.03 & 127.37 \\
HDL-C (mg/dL) & $20-94$ & 43.96 & 8.91 \\
GOT (U/L) & $10-344$ & 28.67 & 16.03 \\
Creatinine (mg/dL) & $0.32-8.47$ & 0.72 & 0.30 \\
Uric acid $(\mathrm{mg} / \mathrm{dL})$ & $0.7-14.1$ & 6.192 & 1.42 \\
\hline
\end{tabular}

After model adjustment of multivariable linear regression with the suggestive SNPs in the Manhattan plot, we found the most significant SNPs associated with microalbuminuria to be rs117180252 ( $\mathrm{p}=0.049$ with CT genotype, located on chromosome 5), rs10105606 ( $\mathrm{p}=0.045$ with CA genotype and $\mathrm{p}=0.040$ with AA genotype, located on chromosome 8), and Affx-31885823 ( $\mathrm{p}=$ 0.040 with CA genotype and $\mathrm{p}=0.048$ with AA genotype, located on chromosome 8). Chromosomes 5 and 8 have been mapped to the RN7SL87P lipoprotein lipase ( $L P L)$ and RPL30P9 gene, respectively (Table 2). Compared to non-microalbuminuria controls, rs10105606 and Affx31885823 allele frequency were significantly higher in MUO participants, with an almost twofold increased risk per copy of the A allele (odds ratio [OR] 1.978; 95\% confidence interval [CI] 1.031-3.796 and OR 1.921; 95\% CI 1.005-3.675) (Table 3). An individual heterozygous for the $\mathrm{T}$ allele in rs117180252 had more than a twofold increased risk of microalbuminuria (OR 2.024; 95\% CI 1.003-4.083), compared with homozygotes for non-risk minor allele $\mathrm{C}$ (Table 3). Apolipoprotein (APO) A5, $A P O B, A P O C 2, A P O C 3$, cholesteryl ester transfer protein $(C E T P)$, and glycosylphosphatidylinositol-anchored highdensity lipoprotein-binding protein 1 (GPIHBP 1$)$ are connected to the $L P L$ gene in the gene-gene interaction network analysis (Figure 3). A flowchart of abdominal obesity and microalbuminuria is shown in Figure 4.

\section{Discussion}

In this study, we have sought the genetic variation of microalbuminuria among 15,300 Han-Chinese in Taiwan. In our sample, $8.66 \%$ of the population was categorized to be with MUO. We have identified three novel microalbuminuria risk genes, namely, RN7SL87P (rs117180252), LPL (rs10105606), and RPL30P9 (Affx-31885823), in the MUO population.

Visceral adipose tissue, which accumulates in the intraabdomen, is strongly related to cardiometabolic disease. ${ }^{20}$ Visceral adipose tissue can secrete adipokines that affect insulin sensitivity and peptides that regulate non-esterified fatty acid and TG metabolism. ${ }^{21}$ Adipokines also modulate inflammatory cytokines, including tumor necrosis factoralpha, monocyte chemoattractant protein-1 (MCP-1), and interleukin-1 beta. $^{20,21}$ The hyperlipolytic state of expanded visceral adipose tissue disrupts normal metabolism, whereas proinflammatory cytokines' excessive circulation contributes to insulin resistance and type 2 diabetes. $^{21}$ 


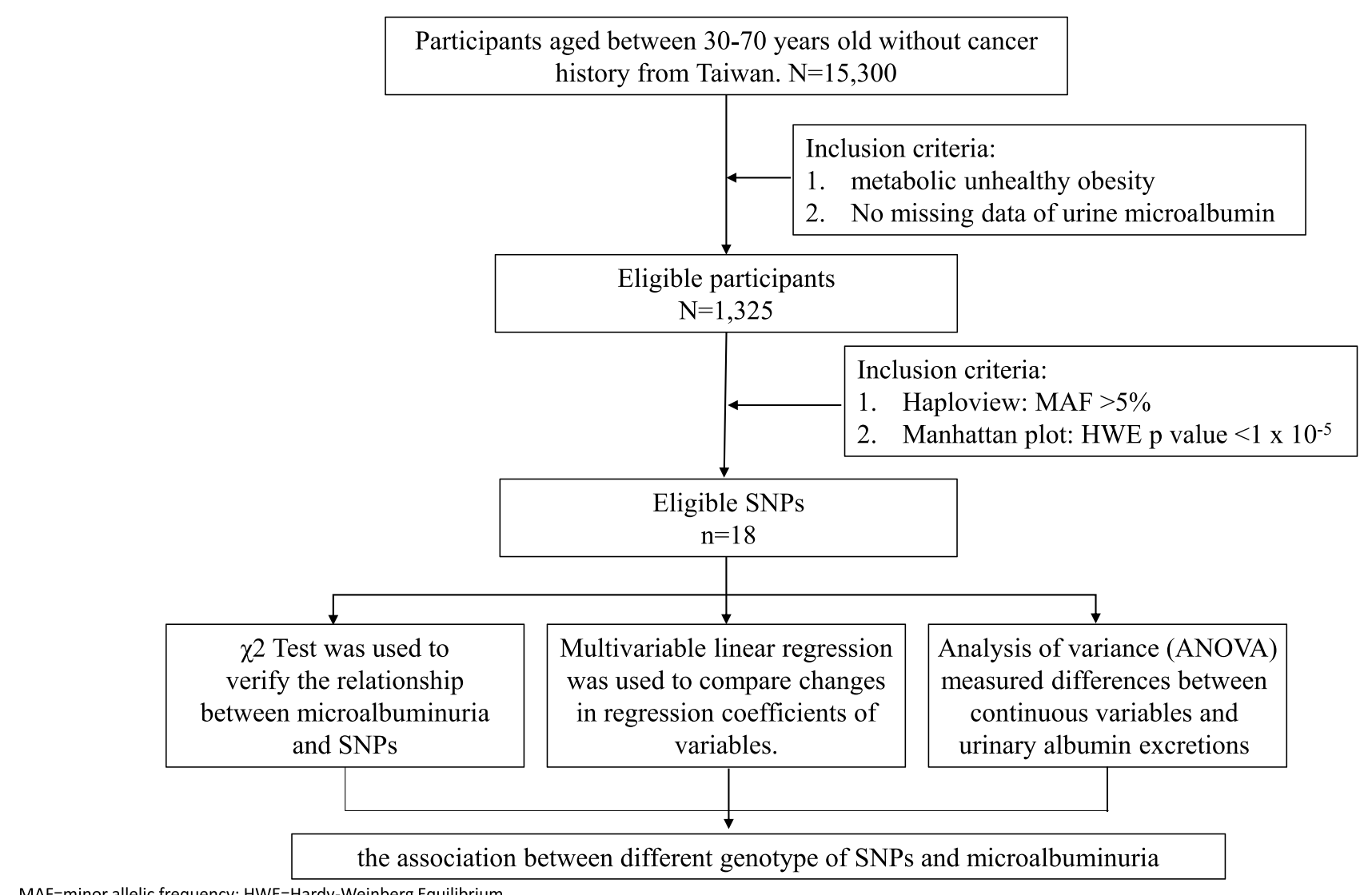

Figure I Flow chart of our research.

Inflammation plays a role in the pathogenesis of microalbuminuria. ${ }^{22-24}$ Inflammation causes microvascular injury of the kidney, particularly the endothelium, leading to vasodilation and microvascular permeability impairment and moderately increased albuminuria. ${ }^{25,26}$ Therefore, microalbuminuria is widely used to predict chronic kidney disease progression and cardiovascular disease. ${ }^{27-29}$ Microalbuminuria is also used to predict the development of renal insufficiency in an asymptomatic proteinuria adult. ${ }^{30}$ Moreover, microalbuminuria is related to ST-T changes of electrocardiography, and both of them have the highest hazard ratio for all-cause mortality. ${ }^{28,31}$ Owing to the above statement, renin-angiotensin-aldosterone system inhibitors have evolved into cornerstones of renal and cardiovascular pharmacotherapies, which are used in the decline of glomerular filtration rate and decrease the risk of the above adverse outcomes. ${ }^{32}$

$L P L$ (rs10105606) was one of the candidate genes of microalbuminuria in MUO patients in this study. $L P L$ belongs to the lipase family and AB hydrolase superfamily, which has coactivators, such as $A P O C 2$ located on the vascular endothelium surface. ${ }^{33}$ The main function of $L P L$ is to hydrolyze TGs of circulating very-low-density lipoproteins (VLDL) and chylomicrons $(\mathrm{CM}){ }^{33}$ It needs to bind to heparin sulfate proteoglycans to maintain its vital function. ${ }^{33} L P L$ is impaired by diabetic dyslipidemia, and its activity is suppressed under an insulin resistance environment. ${ }^{34,35}$ The increased circulation of blood TGs, oxidized low-density lipoproteins (LDLs), and decreased HDL-C enhances the excessive extracellular matrix and macrophage infiltration in the glomeruli and aggravates the vascular and renal cellular dysfunction in the early stage of microalbuminuria and the progression of diabetic nephropathy. ${ }^{36}$ At the same time, statin is involved in the cholesterol synthesis pathway and is used in reducing albuminuria in diabetic nephropathy patients. The mechanism was the inhibitory effect of statin in Rho-kinase and inflammatory pathways. ${ }^{37}$

$L P L$ has known interactions from curated databases and experimentally determined to CETP, GPIHBPI, $A P O C 2$, and $A P O A 5$ in the STRING database. ${ }^{33}$ It also has a co-expression with CETP and GPIHBP $1 .^{33}$ Interestingly, $L P L, A P O C 2, A P O A 5$, and GPIHBP1 are single nucleotide variants in the primary TG-related 


\begin{tabular}{|c|c|c|c|c|c|c|c|c|c|c|c|c|}
\hline 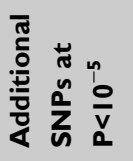 & - & - & 0 & $N$ & $N$ & $N$ & 으 & 으 & 으 & 으 & 으 & 으 \\
\hline 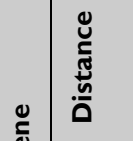 & 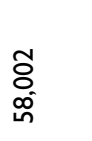 & 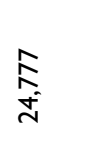 & $\begin{array}{l}\infty \\
0 \\
0 \\
0 \\
\text { m. }\end{array}$ & \begin{tabular}{l}
$\hat{\sim}$ \\
$\underset{\infty}{+}$ \\
\multirow{\infty}{*}{}
\end{tabular} & $\begin{array}{l}\frac{a}{m} \\
\underline{\underline{\omega}}\end{array}$ & $\begin{array}{l}\text { m } \\
= \\
=\end{array}$ & 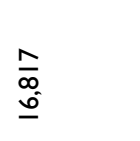 & $\begin{array}{l}\stackrel{\infty}{0} \\
\stackrel{0}{i} \\
\stackrel{m}{m}\end{array}$ & 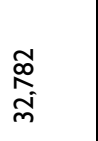 & 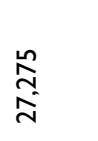 & $\frac{\bar{\alpha}}{\alpha}$ & $\begin{array}{l}\stackrel{\rho}{m} \\
\stackrel{m}{0}\end{array}$ \\
\hline 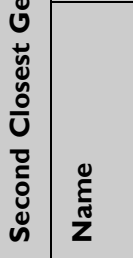 & $\begin{array}{l}\frac{a}{a} \\
\hat{a} \\
\frac{a}{a} \\
a\end{array}$ & 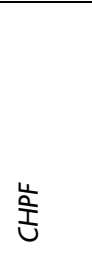 & $\frac{n}{\frac{n}{2}}$ & $\frac{0}{\hat{n}}$ & $\frac{0}{5}$ & $\vec{a}$ & $\underset{\underline{Z}}{\stackrel{y}{Z}}$ & $\begin{array}{l}\bar{\alpha} \\
\hat{N}\end{array}$ & $\begin{array}{l}\bar{\alpha} \\
\stackrel{\bar{\alpha}}{N}\end{array}$ & $\begin{array}{l}\bar{\alpha} \\
\stackrel{\tilde{N}}{N}\end{array}$ & 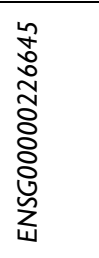 & 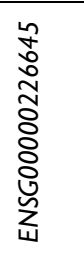 \\
\hline 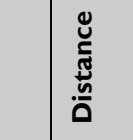 & $\stackrel{\tilde{\sigma}}{\underline{\widetilde{q}}}$ & $\begin{array}{l}\text { 号 } \\
\text { 品 } \\
\text { - }\end{array}$ & $\begin{array}{l}\stackrel{\infty}{\infty} \\
\stackrel{m}{a} \\
\underline{a}\end{array}$ & 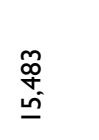 & $\begin{array}{l}\text { : } \\
\text { రీ } \\
0 \\
0\end{array}$ & 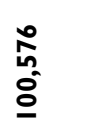 & $\begin{array}{l}\text { o } \\
\text { o } \\
\stackrel{\text { D }}{2}\end{array}$ & $\begin{array}{l}\stackrel{g}{+} \\
=\end{array}$ & $\frac{\mathfrak{n}}{\wedge}$ & $\stackrel{f}{0}$ & 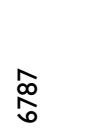 & $\begin{array}{l}\infty \\
\stackrel{+}{0} \\
\stackrel{\circ}{n}\end{array}$ \\
\hline 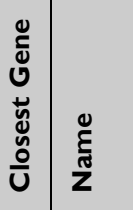 & $\frac{5}{\substack{1 \\
⿱ 亠 䒑}}$ & 离 & 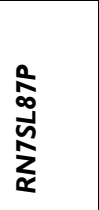 & 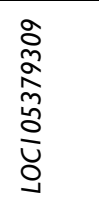 & $\vec{a}$ & 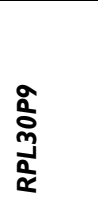 & $\begin{array}{l}\infty \\
\sum_{<}^{\infty} \\
\infty\end{array}$ & 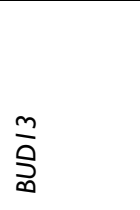 & $\frac{m}{9}$ & $\stackrel{m}{9}$ & 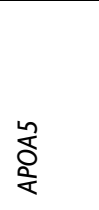 & $\begin{array}{l}\frac{2}{0} \\
\frac{1}{2}\end{array}$ \\
\hline$\frac{\mathscr{x}}{\alpha}$ & $\begin{array}{l}\bar{ָ} \\
\text { ণ }\end{array}$ & స્ત્ & ڤ્̀े & 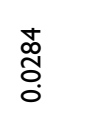 & 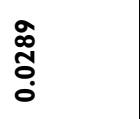 & $\begin{array}{l}\infty \\
\stackrel{0}{0} \\
0 \\
0\end{array}$ & $\underset{\tilde{N}}{\stackrel{\tilde{O}}{0}}$ & $\begin{array}{l}\hat{\sim} \\
\text { ป. } \\
0\end{array}$ & 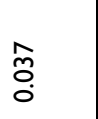 & $\begin{array}{l}\text { } \\
\substack{\infty \\
+\\
0}\end{array}$ & 岕 & $\frac{\nabla}{0}$ \\
\hline $\begin{array}{l}\frac{0}{\pi} \\
\frac{0}{2} \\
0\end{array}$ & 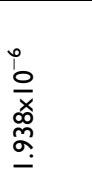 & $\begin{array}{l}\frac{0}{x} \\
\frac{0}{0} \\
\frac{0}{-}\end{array}$ & 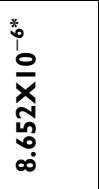 & $\begin{array}{l}\stackrel{0}{x} \\
\stackrel{0}{x} \\
\stackrel{4}{=}\end{array}$ & 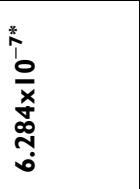 & 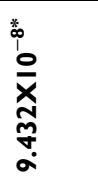 & 柋 & $\begin{array}{l}\hat{0} \\
\frac{0}{x} \\
o \\
\infty \\
-\end{array}$ & $\begin{array}{l}\frac{0}{0} \\
\frac{0}{x} \\
\frac{f}{j}\end{array}$ & 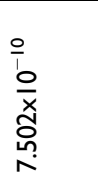 & $\begin{array}{l}\frac{a}{0} \\
\frac{0}{x} \\
\alpha \\
i \\
m\end{array}$ & 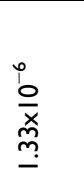 \\
\hline 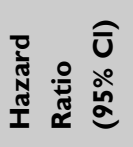 & $\begin{array}{c}\bar{m} \\
\substack{\infty \\
0}\end{array}$ & $\begin{array}{c}\infty \\
\stackrel{\infty}{\infty} \\
\infty \\
0 \\
0\end{array}$ & $\begin{array}{l}\text { N } \\
\infty \\
\stackrel{0}{0} \\
0\end{array}$ & 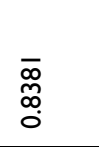 & 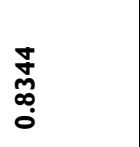 & $\underset{\substack{0 \\
0}}{\tilde{\infty}}$ & $\stackrel{\stackrel{\rho}{m}}{=}$ & $\stackrel{\infty}{\underset{̣}{\underline{~}}}$ & 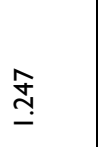 & $\frac{\substack{m \\
\infty \\
\infty \\
0}}{0}$ & $\stackrel{\text { İ }}{\underline{I}}$ & 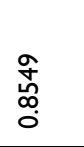 \\
\hline 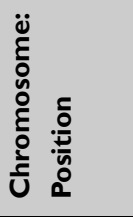 & 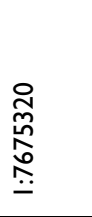 & 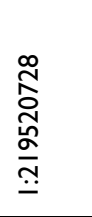 & $\frac{\bar{c}}{\frac{\bar{n}}{N}}$ & $\begin{array}{l}\bar{\alpha} \\
\stackrel{\circ}{\circ} \\
\alpha \\
\stackrel{\infty}{\infty}\end{array}$ & $\begin{array}{l}\hat{\tilde{a}} \\
\frac{\hat{c}}{a} \\
\frac{\alpha}{\alpha}\end{array}$ & 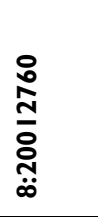 & 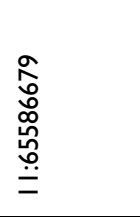 & 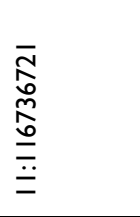 & $\begin{array}{l}\frac{\overline{ }}{0} \\
\frac{1}{\hat{o}} \\
\frac{\bar{\Xi}}{=}\end{array}$ & 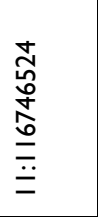 & 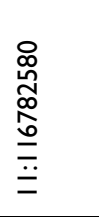 & 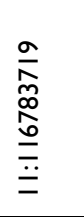 \\
\hline$\frac{\mathrm{L}}{\mathbf{\Sigma}}$ & $\begin{array}{l}\frac{\infty}{0} \\
\frac{m}{0}\end{array}$ & $\frac{a}{0}$ & $\begin{array}{l}\overline{0} \\
\infty \\
0 \\
0\end{array}$ & $\frac{\sigma}{\sigma}$ & $\frac{a}{a}$ & $\frac{5}{\circ}$ & $\begin{array}{l}\stackrel{0}{0} \\
\text { fँ } \\
0\end{array}$ & 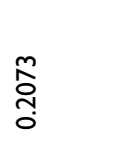 & $\begin{array}{l}\text { ठ̊̀ } \\
\text { Oे }\end{array}$ & 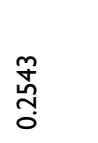 & $\begin{array}{l}\stackrel{\tilde{N}}{\tilde{O}} \\
\text { }\end{array}$ & 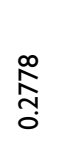 \\
\hline 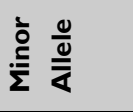 & $\vdash$ & $u$ & $\vdash$ & $u$ & $\varangle$ & $\varangle$ & $\varangle$ & $u$ & $u$ & $u$ & $u$ & $\vdash$ \\
\hline 䒺 & 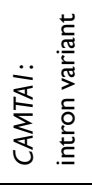 & 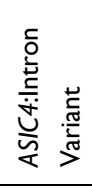 & 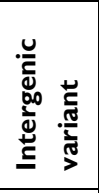 & 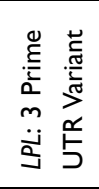 & 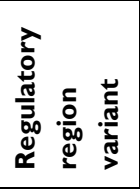 & $\overline{\mathbf{z}}$ & 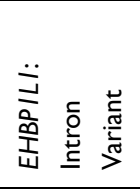 & 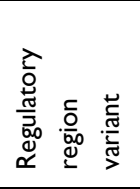 & 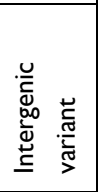 & 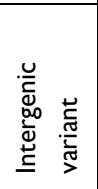 & 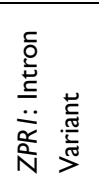 & 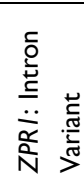 \\
\hline$\sum_{n}^{n}$ & 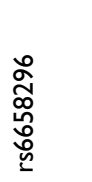 & 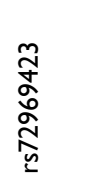 & $\begin{array}{l}\frac{\tilde{N}}{0} \\
\frac{\infty}{n} \\
\frac{\bar{n}}{\underline{n}}\end{array}$ & $\begin{array}{l}\frac{\tilde{\Omega}}{\mathrm{m}} \\
\frac{m}{\underline{n}}\end{array}$ & $\begin{array}{l}\text { 웅 } \\
\text { ㄴํㅇ } \\
\frac{0}{0}\end{array}$ & 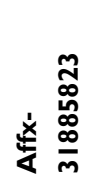 & $\begin{array}{l}\underset{\text { Iิ }}{\text { İ }} \\
\overline{\bar{n}}\end{array}$ & $\begin{array}{l}\bar{o} \\
\stackrel{0}{\infty} \\
\underline{h} \\
\underline{\underline{n}}\end{array}$ & 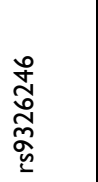 & $\frac{\stackrel{2}{\frac{0}{0}}}{\frac{\overline{0}}{\underline{n}}}$ & 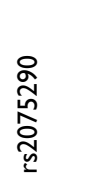 & 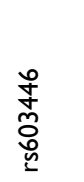 \\
\hline
\end{tabular}




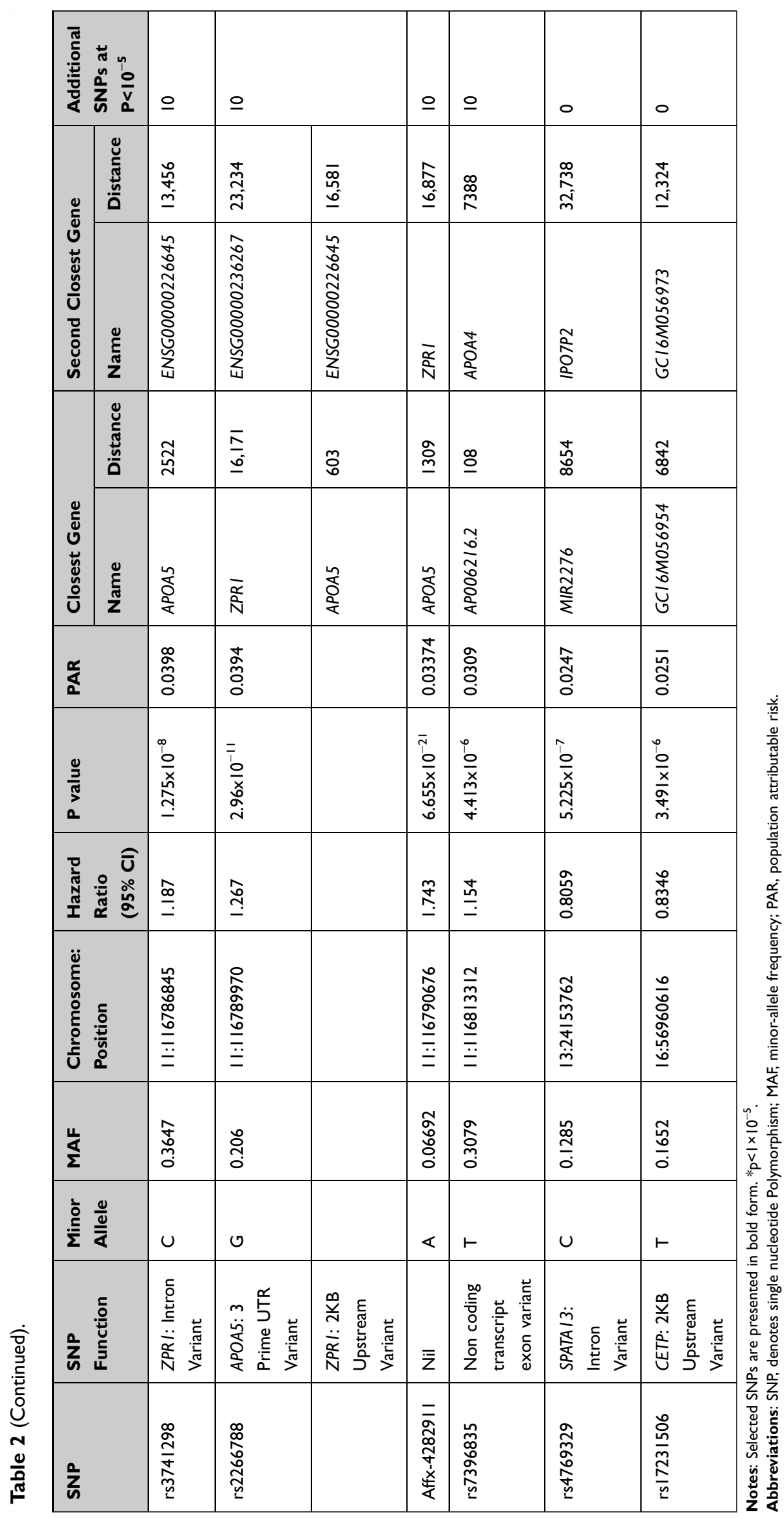




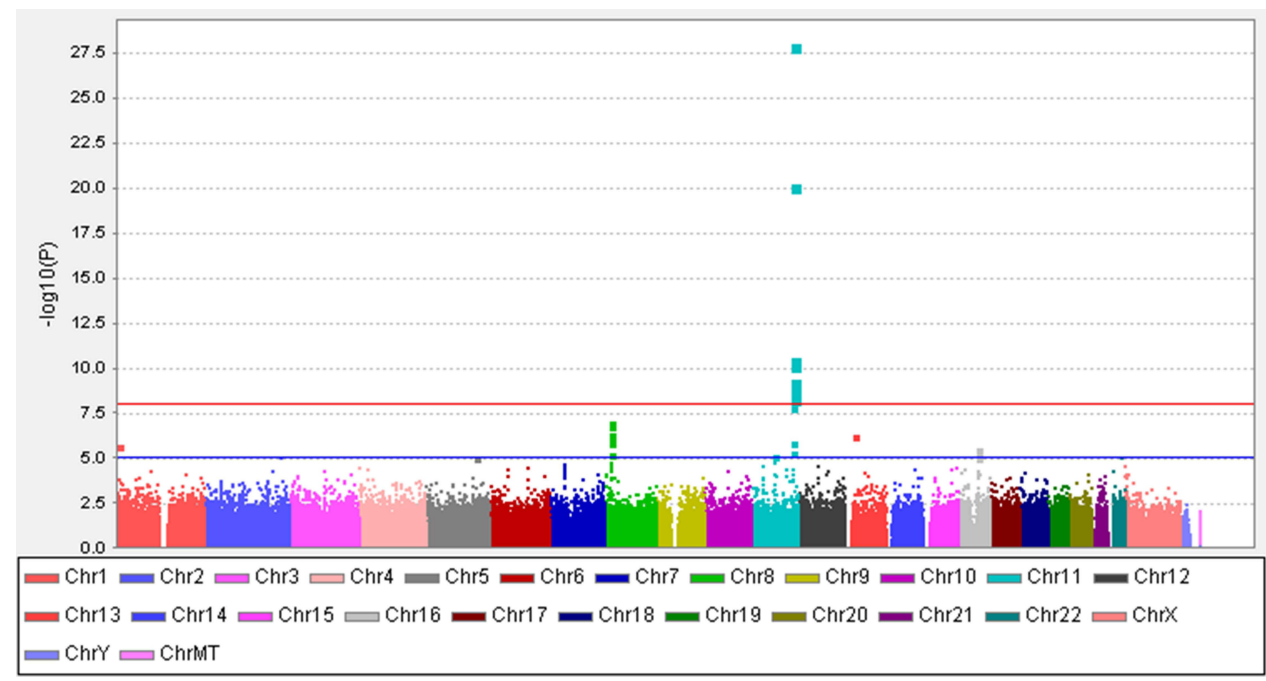

Figure 2 Manhattan plot of the discovery sample.

genes. ${ }^{38}$ CETP belongs to the BPI/LBP/Plunc superfamily, which regulates the transfer of neutral lipids and the reverse transport of excess cholesterol from peripheral tissues to the liver for elimination. ${ }^{39}$ CETP's ability in the transferring of TG to HDL is impaired in diabetic patients. ${ }^{40}$ As a result, HDL cannot send cholesteryl ester to VLDL and further becomes lipid-poor HDL, which the kidney will then filter after being hydrolyzed by hepatic TG lipase or $L P L .^{40}$ GPIHBP1 is LY6/PLAUR domain containing, which has an important role in the lipolytic processing of $\mathrm{CM}^{41}$ It is essential for the transport of $L P L$ into the capillary lumen. ${ }^{41}$ In contrast, $A P O C 2$ and $A P O A 5$ play important roles in lipoprotein metabolism. $A P O C 2$ is the component of four major classes of circulating lipoproteins and acts as an activator of $L P L{ }^{42} A P O A 5$ is a minor apolipoprotein associated with HDL, and the relationship between serum TG and APOA5 gene polymorphism has been demonstrated by a Japanese study. ${ }^{43}$

Dyslipidemia causes lipid nephrotoxicity and also facilitates glomerulosclerosis. ${ }^{44}$ TG-rich lipoprotein receptors (TGRLs) and scavenger receptors are both expressed in podocytes and mesangial cells in glomeruli. ${ }^{45-47}$ TGrich lipoproteins are involved in secreting proinflammatory cytokines, including transforming growth factor (TGF)alpha, TGF-beta, and interleukin 6, which eventually leads to the excessive production of mitochondrial reactive oxygen species induced by the extracellular matrix. ${ }^{48}$ TGRLs also disrupt endothelial cell glycocalyx, which regulates glomerular permeability. ${ }^{44}$ Although scavenger receptors are bound by oxidized LDL, they stimulate
MCP-1 and cause monocyte migration, thus facilitating macrophages to become foam cells and damage renal vasculature. $^{49}$

RN7SL87P (rs117180252) and RPL30P9 (Affx$31885823)$ were the other two candidate genes in this study. However, the genome-wide association study (GWAS) catalog and STRING database ${ }^{33}$ could not find the relation between RN7SL87P and RPL30P9, yet they may have some indirect impact on regulating microalbuminuria and abdominal obesity. Further functional validation is crucial to identify these microalbuminuria-related genes in MUO patients.

To our knowledge, there was a positive association between genetic variants on lipid parameters and cardiovascular disease risk. Moreover, scientist has demonstrated the linear correlations between microalbuminuria, BMI, serum lipids and blood pressure. ${ }^{50}$ Microalbuminuria may be an integrated marker in cardiovascular risk, especially in high BMI and dyslipidemia patients, such as MUO patients in order to prevent hypertension and its subsequent cardiovascular disease risk in those who had significant SNPs associated with microalbuminuria in our study.

This study had several strengths. First, Taiwan Biobank (TWB) is a population-based biobank which has generated whole-genome sequencing and genome-wide SNP of Han Chinese ancestry. Second, it had gone through a long period of ethical, legal, social, and scientific review process with multi-omics genomic data. More informations are available at https://www.twbiobank.org.tw/new_web_en/. Second, to the best of our knowledge, this is the first report of inter- 
Table 3 The Hazard Ratios and Population Attributable Risks Associated with the Minor Allele

\begin{tabular}{|c|c|c|c|c|c|c|c|}
\hline \multirow[t]{2}{*}{ Gene (SNP) } & \multirow[t]{2}{*}{ Genotype } & \multicolumn{2}{|c|}{ Control } & \multicolumn{2}{|c|}{ Microalbuminuria } & \multicolumn{2}{|l|}{ Adjusted Model } \\
\hline & & Count & $\begin{array}{l}\text { Per } \\
\text { cent }\end{array}$ & Count & Per cent & $\beta(95 \% \mathrm{Cl})$ & p value \\
\hline \multirow{3}{*}{ CAMTAI (rs6658296) } & $\mathrm{C} / \mathrm{C}$ & 780 & 76.5 & 239 & 23.5 & Reference & \\
\hline & $\mathrm{C} / \mathrm{T}$ & 227 & 74.2 & 79 & 25.8 & I.I32 (0.839, I.526) & 0.418 \\
\hline & $\mathrm{T} / \mathrm{T}$ & 0 & 0 & 0 & 0 & - & - \\
\hline \multirow[t]{4}{*}{ ASIC4 (rs72969423) } & $\mathrm{A} / \mathrm{A}$ & 768 & 76.3 & 238 & 23.7 & Reference & \\
\hline & $\mathrm{A} / \mathrm{C}$ & 222 & 75.0 & 74 & 25.0 & $1.046(0.771,1.419)$ & 0.771 \\
\hline & $\mathrm{C} / \mathrm{C}$ & 17 & 73.9 & 6 & 26.1 & 1.149 & 0.774 \\
\hline & & & & & & $(0.0445,2.97 I)$ & \\
\hline \multirow{3}{*}{$\begin{array}{l}\text { RN7SL87P } \\
\text { (rsII7I80252) }\end{array}$} & $\mathrm{C} / \mathrm{C}$ & 985 & 76.4 & 305 & 23.6 & Reference & \\
\hline & $\mathrm{C} / \mathrm{T}$ & 22 & 62.9 & 13 & 37.1 & $2.024(1.003,4.083)$ & $0.049 *$ \\
\hline & $\mathrm{T} / \mathrm{T}$ & 0 & 0 & 0 & 0 & - & - \\
\hline \multirow[t]{3}{*}{ LPL (rs|3702) } & $\mathrm{T} / \mathrm{T}$ & 683 & 77.5 & 198 & 22.5 & Reference & \\
\hline & $\mathrm{T} / \mathrm{C}$ & 295 & 73.8 & 105 & 26.3 & I.258 $(0.954,1.659)$ & 0.104 \\
\hline & $\mathrm{C} / \mathrm{C}$ & 29 & 65.9 & 15 & 34.1 & $1.874(0.980,3.584)$ & 0.057 \\
\hline \multirow[t]{3}{*}{ LPL (rs 10105606$)$} & $\mathrm{C} / \mathrm{C}$ & 687 & 77.9 & 195 & 22.1 & Reference & \\
\hline & $\mathrm{C} / \mathrm{A}$ & 292 & 73 & 108 & 27 & $1.326(1.007,1.747)$ & $0.045 *$ \\
\hline & $\mathrm{A} / \mathrm{A}$ & 28 & 65.1 & 15 & 34.9 & $1.978(1.031,3.796)$ & $0.040 *$ \\
\hline \multirow{3}{*}{$\begin{array}{l}\text { RPL30P9 (Affx- } \\
31885823 \text { ) }\end{array}$} & $\mathrm{C} / \mathrm{C}$ & 693 & 77.9 & 197 & 22.1 & Reference & \\
\hline & $\mathrm{C} / \mathrm{A}$ & 285 & 72.9 & 106 & 27.1 & $1.336(1.013,1.763)$ & $0.040 *$ \\
\hline & $\mathrm{A} / \mathrm{A}$ & 29 & 65.9 & 15 & 34.1 & $1.921(1.005,3.675)$ & $0.048 *$ \\
\hline \multirow[t]{3}{*}{ EHBPILI (rsII227229) } & $\mathrm{G} / \mathrm{G}$ & 285 & 77.4 & 83 & 22.6 & Reference & \\
\hline & $\mathrm{G} / \mathrm{A}$ & 498 & 75 & 166 & 25 & I.I $47(0.845, \mathrm{I} .556)$ & 0.380 \\
\hline & $\mathrm{A} / \mathrm{A}$ & 224 & 76.5 & 69 & 23.5 & $1.053(0.728,1.522)$ & 0.784 \\
\hline \multirow[t]{3}{*}{ BUD/3 (rs/55886I) } & $\mathrm{T} / \mathrm{T}$ & 589 & 75.4 & 192 & 24.6 & Reference & \\
\hline & $\mathrm{T} / \mathrm{C}$ & 385 & 77.6 & 111 & 22.4 & $0.898(0.685, I .175)$ & 0.432 \\
\hline & $\mathrm{C} / \mathrm{C}$ & 33 & 68.8 & 15 & 31.3 & $\begin{array}{l}1.3819 \\
(0.719,2.650)\end{array}$ & 0.332 \\
\hline \multirow[t]{3}{*}{ BUD/3 (rs9326246) } & $\mathrm{G} / \mathrm{G}$ & 586 & 75.4 & 191 & 24.6 & Reference & \\
\hline & $\mathrm{G} / \mathrm{C}$ & 385 & 77.6 & 111 & 22.4 & $0.899(0.687,1.178)$ & $0.44 I$ \\
\hline & $\mathrm{C} / \mathrm{C}$ & 36 & 69.2 & 16 & 30.8 & $1.295(0.688,2.438)$ & 0.423 \\
\hline \multirow[t]{3}{*}{ BUD /3 (rs I I2/6I26) } & $\mathrm{A} / \mathrm{A}$ & 602 & 75.9 & 191 & 24.1 & Reference & \\
\hline & $\mathrm{A} / \mathrm{C}$ & 347 & 76.6 & 106 & 23.4 & $0.973(0.739, I .28 I)$ & 0.846 \\
\hline & $\mathrm{C} / \mathrm{C}$ & 58 & 73.4 & 21 & 26.6 & $\mathrm{I} .083(0.634, \mathrm{I} .850)$ & 0.770 \\
\hline \multirow[t]{3}{*}{ ZPRI (rs2075290) } & $\mathrm{T} / \mathrm{T}$ & 557 & 74.8 & 188 & 25.2 & Reference & \\
\hline & $\mathrm{T} / \mathrm{C}$ & 409 & 78.2 & 114 & 21.8 & $0.829(0.634,1.085)$ & 0.172 \\
\hline & $\mathrm{C} / \mathrm{C}$ & 41 & 71.9 & 16 & 28.1 & I.I $64(0.628,2.155)$ & 0.630 \\
\hline \multirow[t]{3}{*}{ ZPRI (rs603446) } & $\mathrm{C} / \mathrm{C}$ & 566 & 76.5 & 174 & 23.5 & Reference & \\
\hline & $\mathrm{C} / \mathrm{T}$ & 385 & 75.9 & 122 & 24.1 & I.032 $(0.789$, I.350) & 0.818 \\
\hline & $\mathrm{T} / \mathrm{T}$ & 56 & 71.8 & 22 & 28.2 & $\mathrm{I} .200(0.702,2.05 \mathrm{I})$ & 0.505 \\
\hline
\end{tabular}

(Continued) 
Table 3 (Continued).

\begin{tabular}{|c|c|c|c|c|c|c|c|}
\hline \multirow[t]{2}{*}{ Gene (SNP) } & \multirow[t]{2}{*}{ Genotype } & \multicolumn{2}{|c|}{ Control } & \multicolumn{2}{|c|}{ Microalbuminuria } & \multicolumn{2}{|l|}{ Adjusted Model } \\
\hline & & Count & $\begin{array}{l}\text { Per } \\
\text { cent }\end{array}$ & Count & Per cent & $\beta(95 \% \mathrm{Cl})$ & p value \\
\hline \multirow[t]{3}{*}{ ZPRI (rs374I298) } & $T / T$ & 362 & 73.3 & 132 & 26.7 & Reference & \\
\hline & $\mathrm{T} / \mathrm{C}$ & 490 & 77.8 & 140 & 22.2 & $0.817(0.619,1.078)$ & 0.153 \\
\hline & $\mathrm{C} / \mathrm{C}$ & 155 & 77.1 & 46 & 22.9 & $0.856(0.579,1.266)$ & 0.436 \\
\hline \multirow[t]{3}{*}{ APOA5/ZPRI (rs2266788) } & $\mathrm{A} / \mathrm{A}$ & 591 & 75.4 & 193 & 24.6 & Reference & \\
\hline & $\mathrm{A} / \mathrm{G}$ & 380 & 77.4 & 111 & 22.6 & $0.907(0.692,1.187)$ & 0.476 \\
\hline & $\mathrm{G} / \mathrm{G}$ & 36 & 72 & 14 & 28 & I.209 $(0.628,2.328)$ & 0.570 \\
\hline \multirow[t]{3}{*}{ APOA5 (Affx-42829II) } & $\mathrm{C} / \mathrm{C}$ & 822 & 75.9 & 261 & 24.1 & Reference & \\
\hline & $\mathrm{C} / \mathrm{A}$ & 179 & 76.5 & 55 & 23.5 & $0.950(0.679,1.330)$ & 0.766 \\
\hline & $\mathrm{A} / \mathrm{A}$ & 6 & 75 & 2 & 25 & I.050 (0.209,5.29I) & 0.953 \\
\hline \multirow[t]{3}{*}{ AP0062I6.2 (rs7396835) } & $\mathrm{C} / \mathrm{C}$ & 452 & 75.5 & 147 & 24.5 & Reference & \\
\hline & $\mathrm{C} / \mathrm{T}$ & 449 & 77.3 & 132 & 22.7 & $0.886(0.674, I .163)$ & 0.383 \\
\hline & $\mathrm{T} / \mathrm{T}$ & 106 & 73.1 & 39 & 26.9 & $1.186(0.782,1.800)$ & 0.422 \\
\hline \multirow[t]{3}{*}{ SPATA I 3 (rs4769329) } & $T / T$ & 764 & 76.3 & 237 & 23.7 & Reference & \\
\hline & $\mathrm{T} / \mathrm{C}$ & 229 & 74.4 & 79 & 25.6 & I.098 (0.8I4,I.48I) & $0.54 I$ \\
\hline & $\mathrm{C} / \mathrm{C}$ & 14 & 87.5 & 2 & 12.5 & $0.478(0.107,2.131)$ & 0.333 \\
\hline \multirow[t]{3}{*}{ CETP (rs|723I506) } & $\mathrm{C} / \mathrm{C}$ & 750 & 76.8 & 227 & 23.2 & Reference & \\
\hline & $\mathrm{C} / \mathrm{T}$ & 239 & 74.7 & 81 & 25.3 & I.I I $4(0.828$, I.498) & 0.476 \\
\hline & $T / T$ & 18 & 64.3 & 10 & 35.7 & I.570 $(0.684,3.603)$ & 0.287 \\
\hline
\end{tabular}

Notes: Significant SNPs are presented in bold form. ${ }^{*} \mathrm{p}<0.05$.

individual differences of microalbuminuria in MUO patients by using TWB. In contrast, our study has few limitations. First, we did not identify hematuria, red blood cell casts, white blood cell casts, glucosuria, and lipiduria in the same urine sediment sample. However, they were supposed to be healthy persons in the absence of infection, kidney disease, diabetes mellitus, systemic autoimmune disease, and malignancy. Second, we did not rule out transient proteinuria from vigorous exercise and orthostatic proteinuria in male participants. However, it was uncommon in adults older than 30 years, and in our study, there was only a part of participants in the range between 20 and 30 years old.
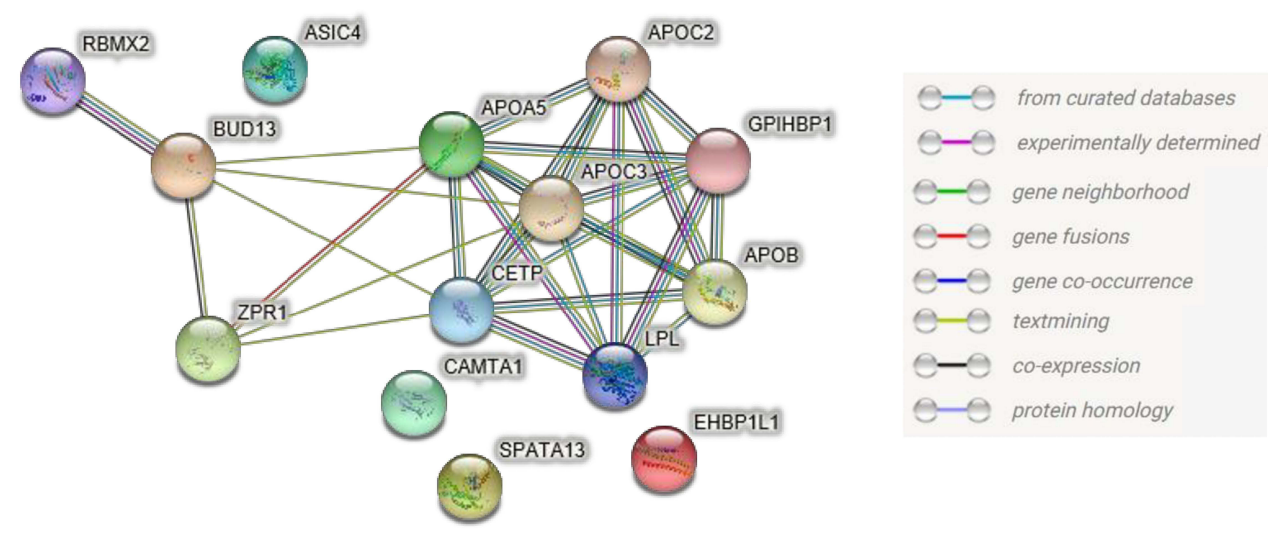

Figure 3 Protein-protein interactions (PPI) network of LPL connected them to lipid-related genes such as CETP, APOA5, and etc. Network nodes represented proteins, and filled nodes represented with known or predicted 3D structure. While the edge represented the interactions between the nodes. Different color of line indicated different type of interactions, which the associations were meant to be specific and meaningful. These proteins jointly contributed to a shared function and were not necessarily mean they were physical binding each other but represented functional interactions. The figure was plotted by STRING. 


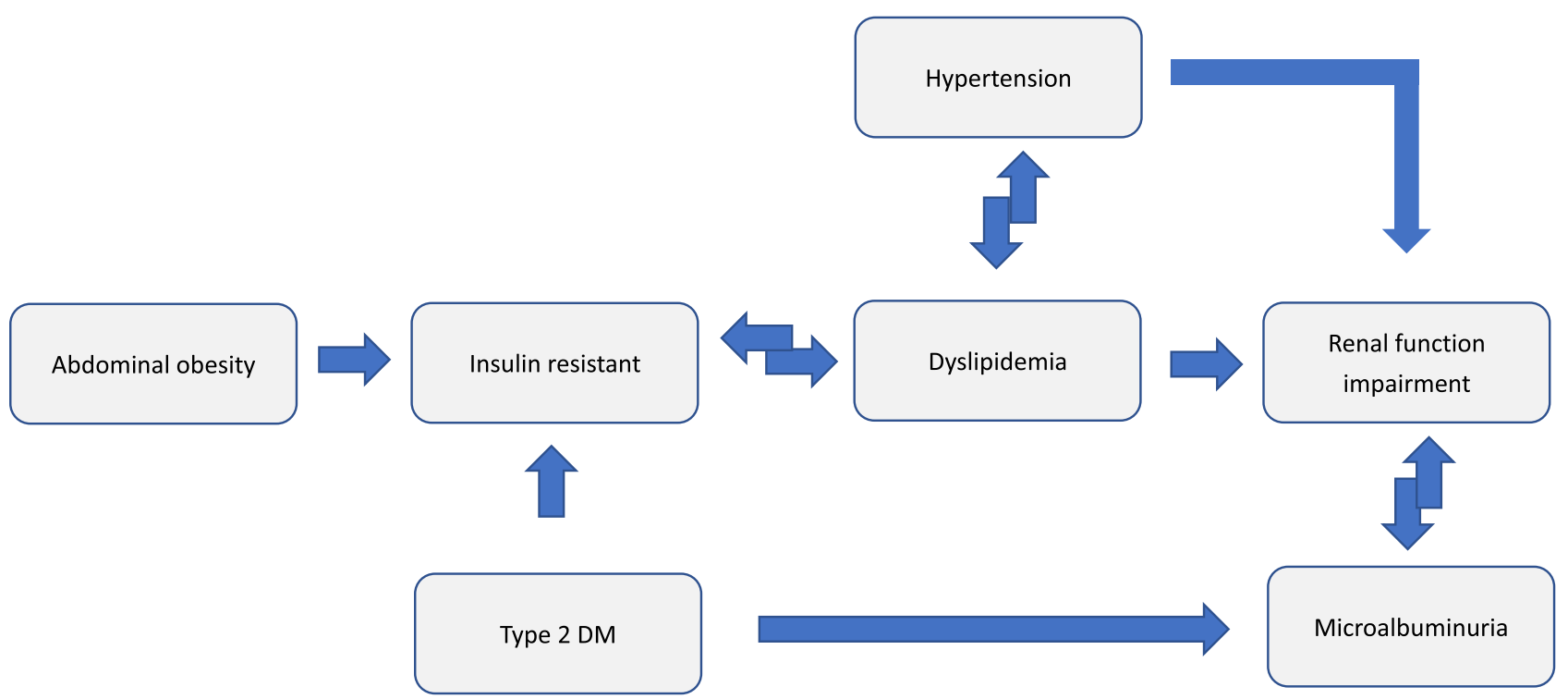

Figure 4 Vicious cycle of metabolic syndrome and microalbuminuria.

In conclusion, we identified novel microalbuminuria risk variants that may participate in lipid metabolism by performing GWAS. Our findings suggest the possible associations between abdominal obesity and microalbuminuria. Although isolated non-nephrotic proteinuria was an indolent course, it may establish a certain degree of glomerulus injury and eventually develop renal dysfunction. Therefore, screening for proteinuria annually among MUO patients may be considered as it is cost effective.

\section{Abbreviations}

MUO, metabolically unhealthy obesity; SNP, Single nucleotide polymorphisms; LPL, Lipoprotein lipase; BMI, body mass index; TGs, triglycerides; HDL-C, high-density lipoprotein cholesterol; FTO, fat mass and obesity-associated; CKD, chronic kidney disease; TWB, Taiwan Biobank; GOT, Glutamic Oxaloacetic Transaminase; LD, linkage disequilibrium; PCA, principal component analysis; HWE, HardyWeinberg equilibrium; CETP, cholesteryl ester transfer protein; GPIHBP1, glycosylphosphatidylinositol-anchored highdensity lipoprotein-binding protein 1; MCP-1, monocyte chemoattractant protein-1; VLDL, very-low-density lipoproteins; CM, chylomicrons; LDLs, low-density lipoproteins; TGRLs, TG-rich lipoprotein receptors; TGF, transforming growth factor; GWAS, Genome-wide association study.

\section{Ethical Approval}

This article does not contain any studies with human participants or animals performed by any of the authors.
This study was conducted in accordance with the Declaration of Helsinki.

\section{Informed Consent}

Written informed consent was obtained from all individual participants included in the study.

\section{Funding}

This research did not receive any specific grant from any funding agency in the public, commercial or not-for-profit sector.

\section{Disclosure}

The authors declare no competing interests.

\section{References}

1. Alberti K, Eckel RH, Grundy SM, et al. Harmonizing the metabolic syndrome: a joint interim statement of the international diabetes federation task force on epidemiology and prevention; national heart, lung, and blood institute; American heart association; world heart federation; international atherosclerosis society; and international association for the study of obesity. Circulation. 2009;120(16):1640-1645. doi:10.1161/CIRCULATIONAHA.109.1 92644

2. Chang B-C-C, Hwang L-C, Huang W-H. Positive association of metabolic syndrome with a single nucleotide polymorphism of Syndecan-3 (rs2282440) in the Taiwanese population. Int J Endocrinol. 2018;2018. doi: $10.1155 / 2018 / 9282598$

3. Povel CM, Boer JMA, Onland-Moret NC, Dollé MET, Feskens EJM, van der Schouw YT. Single nucleotide polymorphisms (SNPs) involved in insulin resistance, weight regulation, lipid metabolism and inflammation in relation to metabolic syndrome: an epidemiological study. Cardiovasc Diabetol. 2012;11:133. doi:10.1186/1475-2840$11-133$ 
4. Saldaña-Alvarez Y, Salas-Martínez MG, García-Ortiz H, et al. Gender-dependent association of FTO polymorphisms with body mass index in Mexicans. PLoS One. 2016;11(1):e0145984. doi:10.1371/journal.pone. 0145984

5. Consortium WTCC. Genome-wide association study of 14,000 cases of seven common diseases and 3000 shared controls. Nature. 2007;447(7145):661

6. Huang W-H, Hwang L-C, Chan H-L, Lin H-Y, Lin Y-H. Study of seven single-nucleotide polymorphisms identified in East Asians for association with obesity in a Taiwanese population. BMJ Open. 2016;6(8):e011713. doi:10.1136/bmjopen-2016-011713

7. Berezina A, Belyaeva O, Berkovich O, et al. Prevalence, risk factors, and genetic traits in metabolically healthy and unhealthy obese individuals. Biomed Res Int. 2015;2015:1-9. doi:10.1155/2015/548734

8. Stevens PE, Levin A. Evaluation and management of chronic kidney disease: synopsis of the kidney disease: improving global outcomes 2012 clinical practice guideline. Ann Intern Med. 2013;158(11):825830. doi:10.7326/0003-4819-158-11-201306040-00007

9. Steemburgo T, de Azevedo MJ, Gross JL, Milagro F, Campión J, Martínez JA. The rs7204609 polymorphism in the fat mass and obesity-associated gene is positively associated with central obesity and microalbuminuria in patients with type 2 diabetes from Southern Brazil. J Ren Nutr. 2012;22(2):228-236. doi:10.1053/j.jrn.2011.03.004

10. Fan C-T, Lin J-C, Lee C-H. Taiwan Biobank: a project aiming to aid Taiwan's transition into a biomedical island. Pharmacogenomics. 2008;9:235-246. doi:10.2217/14622416.9.2.235

11. Chen C-H, Yang J-H, Chiang CW, et al. Population structure of Han Chinese in the modern Taiwanese population based on 10,000 participants in the Taiwan Biobank project. Hum Mol Genet. 2016;25 (24):5321-5331. doi:10.1093/hmg/ddw346

12. Hannon BA, Edwards CG, Thompson SV, et al. Genetic variants in lipid metabolism pathways interact with diet to influence blood lipid concentrations in adults with overweight and obesity. Lifestyle Genom. 2020;13(6):155-163. doi:10.1159/000507021

13. Jasim AA, Al-Bustan SA, Al-Kandari W, Al-Serri A, AlAskar H. Sequence analysis of APOA5 among the Kuwaiti population identifies association of rs2072560, rs2266788, and rs662799 with TG and VLDL levels. Front Genet. 2018;9:112. doi:10.3389/fgene.2018.00112

14. Villard EF, Eik P, Frisdal E, et al. Genetic determination of plasma cholesterol efflux capacity is gender-specific and independent of HDL-cholesterol levels. Arterioscler Thromb Vasc Biol. 2013;33 (4):822-828. doi:10.1161/ATVBAHA.112.300979

15. Shen Y, Xi B, Zhao X, et al. Common genetic variants associated with lipid profiles in a Chinese pediatric population. Hum Genet. 2013;132(11):1275-1285. doi:10.1007/s00439-013-1332-1

16. Aung LH, Yin RX, Wu DF, Wang W, Wu JZ, Liu CW. Sex-specific association of the zinc finger protein 259 rs2075290 polymorphism and serum lipid levels. Int $J$ Med Sci. 2014;11(5):471-478. doi:10.7150/ijms.8489

17. Gombojav B, Lee SJ, Kho M, Song YM, Lee K, Sung J. Multiple susceptibility loci at chromosome $11 \mathrm{q} 23.3$ are associated with plasma triglyceride in East Asians. J Lipid Res. 2016;57(2):318-324. doi:10.1194/jlr.P063461

18. Guan F, Niu Y, Zhang T, et al. Two-stage association study to identify the genetic susceptibility of a novel common variant of rs2075290 in ZPR1 to type 2 diabetes. Sci Rep. 2016;6(1):29586. doi:10.1038/srep29586

19. Brautbar A, Covarrubias D, Belmont J, et al. Variants in the APOA5 gene region and the response to combination therapy with statins and fenofibric acid in a randomized clinical trial of individuals with mixed dyslipidemia. Atherosclerosis. 2011;219(2):737-742. doi:10.1016/j. atherosclerosis.2011.08.015

20. Miazgowski T, Krzyżanowska-świniarska B, Dziwura-Ogonowska J, Widecka K. The associations between cardiometabolic risk factors and visceral fat measured by a new dual-energy X-ray absorptiometry-derived method in lean healthy Caucasian women. Endocrine. 2014;47(2):500-505. doi:10.1007/s12020-014-0180-7
21. Guilherme A, Virbasius JV, Puri V, Czech MP. Adipocyte dysfunctions linking obesity to insulin resistance and type 2 diabetes. Nat Rev Mol Cell Biol. 2008;9(5):367-377. doi:10.1038/nrm2391

22. Yudkin J, Forrest R, Jackson C. Microalbuminuria as predictor of vascular disease in non-diabetic subjects: Islington diabetes survey. Lancet. 1988;332(8610):530-533. doi:10.1016/S0140-6736(88) 92657-8

23. Lee SH, Lee TW, Ihm CG, Kim MJ, Woo JT, Chung JH. Genetics of diabetic nephropathy in type $2 \mathrm{DM}$ : candidate gene analysis for the pathogenic role of inflammation. Nephrology. 2005;10:S32-S36. doi:10.1111/j.1440-1797.2005.00454.x

24. Libby P, Theroux P. Pathophysiology of coronary artery disease. Circulation. 2005;111(25):3481-3488.

25. Clausen P, Jensen J, Jensen G, Borch-Johnsen K, Feldt-Rasmussen B. Elevated urinary albumin excretion is associated with impaired arterial dilatory capacity in clinically healthy subjects. Circulation. 2001;103(14):1869-1874. doi:10.1161/01.CIR.103.14.1869

26. Stehouwer CA, Zeldenrust G, den Ottolander GH, Hackeng W, Donker A, Nauta J. Urinary albumin excretion, cardiovascular disease, and endothelial dysfunction in non-insulin-dependent diabetes mellitus. lancet. 1992;340(8815):319-323. doi:10.1016/0140-6736 (92)91401-S

27. Roest M, Banga JD, Janssen WM, et al. Excessive urinary albumin levels are associated with future cardiovascular mortality in postmenopausal women. Circulation. 2001;103(25):3057-3061. doi:10.116 $1 / \mathrm{hc} 2501.091353$

28. Klausen K, Borch-Johnsen K, Feldt-Rasmussen B, et al. Very low levels of microalbuminuria are associated with increased risk of coronary heart disease and death independently of renal function, hypertension, and diabetes. Circulation. 2004;110(1):32-35. doi:10.1161/01.CIR.0000133312.96477.48

29. Arnlov J, Evans JC, Meigs JB, et al. Low-grade albuminuria and incidence of cardiovascular disease events in nonhypertensive and nondiabetic individuals: the Framingham Heart Study. Circulation. 2005;112 (7):969-975. doi:10.1161/CIRCULATIONAHA.105.538132

30. Yamagata K, Yamagata Y, Kobayashi M, Koyama A. A long-term follow-up study of asymptomatic hematuria and/or proteinuria in adults. Clin Nephrol. 1996;45(5):281-288.

31. Diercks GF, Hillege HL, van Boven AJ, et al. Microalbuminuria modifies the mortality risk associated with electrocardiographic STT segment changes. J Am Coll Cardiol. 2002;40(8):1401-1407. doi:10.1016/S0735-1097(02)02165-4

32. Mullens W, Damman K, Testani JM, et al. Evaluation of kidney function throughout the heart failure trajectory-a position statement from the Heart Failure Association of the European Society of Cardiology. Eur $J$ Heart Fail. 2020;22(4):584-603. doi:10.1002/ejhf.1697

33. Singh AN, Gasman B. Disentangling the genetics of sarcopenia: prioritization of NUDT3 and KLF5 as genes for lean mass \& HLADQB1-AS1 for hand grip strength with the associated enhancing SNPs \& a scoring system. BMC Med Genet. 2020;21(1):1-11. doi:10.1186/s12881-020-0977-6

34. Rutledge JC, Ng KF, Aung HH, Wilson DW. Role of triglyceride-rich lipoproteins in diabetic nephropathy. Nat Rev Nephrol. 2010;6 (6):361. doi:10.1038/nrneph.2010.59

35. Pollare T, Vessby B, Lithell H. Lipoprotein lipase activity in skeletal muscle is related to insulin sensitivity. Arterioscler Thromb. 1991;11 (5):1192-1203. doi:10.1161/01.ATV.11.5.1192

36. Svensson M, Eriksson JW. Insulin resistance in diabetic nephropathy - cause or consequence? Diabetes Metab Res Rev. 2006;22(5):401410. doi:10.1002/dmrr.648

37. Mooradian AD, Haas MJ, Wehmeier KR, Wong NC. Obesity-related changes in high-density lipoprotein metabolism. Obesity. 2008;16 (6):1152-1160. doi:10.1038/oby.2008.202

38. Dron JS, Wang J, Cao H, et al. Severe hypertriglyceridemia is primarily polygenic. J Clin Lipidol. 2019;13(1):80-88. doi:10.1016/ j.jacl.2018.10.006 
39. Barter PJ, Brewer HB Jr, Chapman MJ, Hennekens CH, Rader DJ, Tall AR. Cholesteryl ester transfer protein: a novel target for raising HDL and inhibiting atherosclerosis. Arterioscler Thromb Vasc Biol. 2003;23(2):160-167. doi:10.1161/01.ATV.0000054658.91146.64

40. Nieuwdorp M, Meuwese MC, Mooij HL, et al. Measuring endothelial glycocalyx dimensions in humans: a potential novel tool to monitor vascular vulnerability. J Appl Physiol. 2008;104(3):845-852. doi:10.1152/japplphysiol.00440.2007

41. Davies BS, Beigneux AP, Barnes RH II, et al. GPIHBP1 is responsible for the entry of lipoprotein lipase into capillaries. Cell Metab. 2010;12(1):42-52. doi:10.1016/j.cmet.2010.04.016

42. Jong MC, Hofker MH, Havekes LM. Role of ApoCs in lipoprotein metabolism: functional differences between ApoC1, ApoC2, and ApoC3. Arterioscler Thromb Vasc Biol. 1999;19(3):472-484. doi:10.1161/01.ATV.19.3.472

43. Yamada Y, Ichihara S, Kato K, et al. Genetic risk for metabolic syndrome: examination of candidate gene polymorphisms related to lipid metabolism in Japanese people. J Med Genet. 2008;45(1):2228. doi:10.1136/jmg.2007.052415

44. Kawanami D, Matoba K, Utsunomiya K. Dyslipidemia in diabetic nephropathy. Ren Replace. 2016;2(1):1-9. doi:10.1186/s41100-016-0028-0
45. Wheeler D, Fernando R, Gillett M, et al. Characterisation of the binding of low-density lipoproteins to cultured rat mesangial cells. Nephrol Dial Transplant. 1991;6(10):701-708. doi:10.1093/ndt/6.10.701

46. Gröne H-J, Walli AK, Gröne E, Krämer A, Clemens MR, Seidel D. Receptor mediated uptake of apo B and apo E rich lipoproteins by human glomerular epithelial cells. Kidney Int. 1990;37(6):14491459. doi:10.1038/ki.1990.135

47. Quaschning T, Königer M, Krämer-Guth A, et al. Receptor-mediated lipoprotein uptake by human glomerular cells: comparison with skin fibroblasts and HepG2 cells. Nephrol Dial Transplant. 1997;12 (12):2528-2536. doi:10.1093/ndt/12.12.2528

48. Nishida Y, Oda H, Yorioka N. Effect of lipoproteins on mesangial cell proliferation. Kidney Int. 1999;56:S51-S53. doi:10.1046/j.15231755.1999.07113.x

49. Nosadini R, Tonolo G. Role of oxidized low density lipoproteins and free fatty acids in the pathogenesis of glomerulopathy and tubulointerstitial lesions in type 2 diabetes. Nutr Metab Cardiovasc. 2011;21 (2):79-85. doi:10.1016/j.numecd.2010.10.002

50. Leoncini G, Sacchi G, Ravera M, et al. Microalbuminuria is an integrated marker of subclinical organ damage in primary hypertension. J Hum Hypertens. 2002;16(6):399-404. doi:10.1038/sj.jhh.1001408
Journal of Inflammation Research

\section{Publish your work in this journal}

The Journal of Inflammation Research is an international, peerreviewed open-access journal that welcomes laboratory and clinical findings on the molecular basis, cell biology and pharmacology of inflammation including original research, reviews, symposium reports, hypothesis formation and commentaries on: acute/chronic inflammation; mediators of inflammation; cellular processes; molecular

\section{Dovepress}

mechanisms; pharmacology and novel anti-inflammatory drugs; clinical conditions involving inflammation. The manuscript management system is completely online and includes a very quick and fair peerreview system. Visit http://www.dovepress.com/testimonials.php to read real quotes from published authors. 